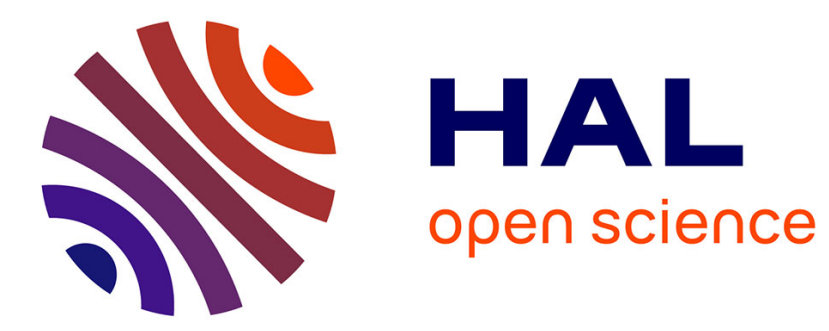

\title{
Auto-Oscillations and Chaos for Microwave Signals in Ferrite Single Crystals
}

H. Benner

\section{To cite this version:}

H. Benner. Auto-Oscillations and Chaos for Microwave Signals in Ferrite Single Crystals. Journal de Physique IV Proceedings, 1997, 07 (C1), pp.C1-383-C1-386. 10.1051/jp4:19971156 . jpa-00254801

\section{HAL Id: jpa-00254801 https://hal.science/jpa-00254801}

Submitted on 1 Jan 1997

HAL is a multi-disciplinary open access archive for the deposit and dissemination of scientific research documents, whether they are published or not. The documents may come from teaching and research institutions in France or abroad, or from public or private research centers.
L'archive ouverte pluridisciplinaire HAL, est destinée au dépôt et à la diffusion de documents scientifiques de niveau recherche, publiés ou non, émanant des établissements d'enseignement et de recherche français ou étrangers, des laboratoires publics ou privés. 


\title{
Auto-Oscillations and Chaos for Microwave Signals in Ferrite Single Crystals
}

\author{
H. Benner
}

Institut für Festkörperphysik and SFB 185, TH Darmstadt, Hochschulstr. 6, 64289 Darmstadt, Germany

\begin{abstract}
The recent progress of nonlinear dynamics has stimulated a renaissance of high-power ferromagnetic resonance experiments on ferrites, both under the aspects of basic research and application. We report on resonance experiments in yttrium iron garnet spheres probing chaotic scenarios above the first-order Suhl threshold. Different techniques have been proposed to change the observed irregular auto-oscillations of magnetization into regular motion. The delayed feedback control method of Pyragas is studied both in theory and experimental application.
\end{abstract}

\section{INTRODUCTION}

Parametric excitation of spin waves is observed in high power FMR experiments when the amplitude of the pumping microwave field exceeds a certain instability threshold well-known as the first- or second-order Suhl instability. Parametrically excited spin waves show a variety of nonlinear phenomena, such as low-frequency autooscillations, quasiperiodicity, mode-locking, intermittency and chaos, which result from nonlinear couplings between thern and have been widely studied for more than a decade [1].

In spite of the various efforts to observe, to analyse and to understand the chaotic behaviour of nonlinear systems, irregular and unpredictable motion is generally not desired in practical applications. Thus, the 'control' of chaos has become subject of intensified research recently. Different strategies have been proposed how to change the irregular into regular motion: Simple non-feedback methods are generally based on the indirect change of some system parameter and require larger controlling power. More sophisticated feedback methods aim at the stabilisation of existing unstable periodic orbits. Since the latter techniques make use of the intrinsic properties of the underlying chaotic attractor they generally can be run with very small controlling power, but require detailed information on the system and are rather intricate to perform.

The specific challenge of analysing and controlling real spin systems is mainly related to their complex dynamic behaviour and to the fast time scale of regular and irregular auto-oscillations, which is in the order of microseconds. Therefore, sophisticated concepts which are based on real-time numerical analysis are generally too slow and can only be applied in a modified way [2].

\section{HIGH POWER FERROMAGNETIC RESONANCE EXPERIMENTS}

Suhl's first-order spin-wave instability is characterized by the decay of the externally driven uniform mode into two spin waves of half the pumping frequency $\omega_{k}=\omega_{\mathrm{p}} / 2$ and opposite wave vectors $(k,-k)$ according to the conservation of energy and quasi-momentum. This instability can either be observed off resonance (i.e. with the pumping frequency far away from the usual ferromagnetic resonance, $\omega_{\mathrm{p}} \neq \omega_{0}$ ) as a subsidiary absorption, or directly on the FMR line $\left(\omega_{\mathrm{p}}=\omega_{0}\right)$ within the coincidence regime. Profiting by the resonance amplification of the FMR mode, experiments in the coincidence regime require much less microwave power to reach the threshold (typically some $10 \mu \mathrm{W}$ for high-quality YIG samples).

In view of such small thresholds, high power FMR experiments, in principle, can be performed with a conventional ESR spectrometer. We have studied the subsidiary absorption at $9.26 \mathrm{GHz}$ using a bimodal transmission-type cavity of quality factor 3000. In this case the first-order instability shows up as an additional absorption structure in lower field, which is well separated from the main resonance. With increasing microwave power this structure shows a drastic broadening accompanied by auto-oscillations and sequences of bifurcations. We have systematically analysed the dynamic behaviour of the subsidiary absorption signal at room temperature and fixed pumping frequency, as presented in Fig. 1. The lower line shows the dependence of the Suhl threshold on $H$ 


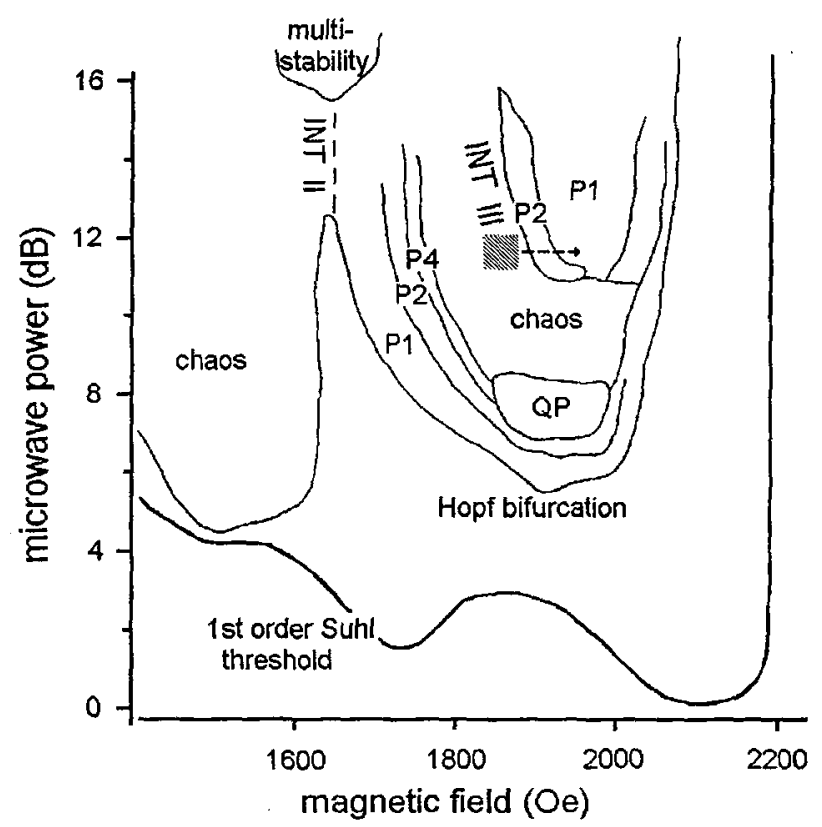

Figure 1: Bifurcation map for subsidiary absorption. The lower line indicates the Suhl threshold, the next upper line ('Hopf bifurcation') the onset of auto-oscillations. QP: quasiperiodic behaviour; P2, P4: period doubling bifurcations; INT II, III; intermittency of Pomeau-Manneville type II, III; dashed area: marginal chaos, application of control.

(the so-called butterfly curve). The bifurcation line above indicates the onset of auto-oscillations. The steep increase of the threshold at $2.2 \mathrm{kOe}$ corresponds to the fact that the bottom of the spin-wave band becomes larger than $\omega_{\mathrm{p}} / 2$ and the parametric process is no longer effective.

Resonant pumping of both the uniform FMR mode and a spin-wave pair $\left(\omega_{\mathrm{p}}=\omega_{0}=2 \omega_{\mathrm{k}}\right)$ is possible in the frequency range from 1.8 to $3.4 \mathrm{GHz}$. Within this coincidence regime the first-order threshold shows up as a sharp and asymmetric break at the top of the main resonance line accompanied by auto-oscillations. The break becomes broader with increasing input power and may be followed by further breaks resulting in a complex multistability, which is connected with a variety, of auto-oscillations. Details have been presented in the literature [3,4] and have been explained in terms of a model including the specific properties of discrete magnetostatic modes in the parametric process, which result in a novel coupling mechanism [3]. According to this model the sudden jumps from one level to the other are induced by the nonlinear coupling or decoupling of certain spin-wave modes.

\section{OBSERVED ROUTES TO CHAOS}

For systematic analysis of the observed auto-oscillations, a large number of time series - up to 16000 data points each - was recorded on variation of $P_{\text {in }}, H$, or other control parameters. The corresponding power spectra were obtained by Fourier transformation, and their strongest spectral components were plotted versus the parameter under variation. The obtained 'maps of auto-oscillations' are useful for classifying the observed routes to chaos.

As a general result, we found that a global correspondence to one of the well-known scenarios of Feigenbaum, Ruelle-Takens-Newhouse or Pomeau-Manneville [5] does not occur, but a variety of parts from all of them. This obviously corresponds to the fact that the nonlinearities in real systems are more complicated and based on a larger number of internal degrees of freedom than represented by the simple models where these standard routes have been derived from. The physical meanirig of the degrees of freedom is probably that of specific eigenmodes or a collective motion of several of them.

Often quasiperiodicity was observed with up to three fundamental frequencies which are typically in the range of 100 to $500 \mathrm{kHz}$. Close above the Suhl threshold a first Hopf bifurcation changes the stationary state into an oscillatory state ('limit cycle'). On further increase of the input power a second fundamental frequency occurs corresponding to a second Hopf bifurcation - together with several sum and difference frequencies of harmonics. Both oscillation frequencies behave independently on variation of the input power, which indicates that the 
attractor is a 2-torus. Finally the spin system changes over to chaos. We also found experimental examples where a third fundamental frequency occurred for an extended parameter range. We suppose that the chaotic behaviour results from a sudden increase of the number of coupled modes, which is related to a global symmetry-breaking bifurcation [2].

A period-doubling route was observed up to period 8, but occurred rather seldom. More often, only a single period doubling occurred, remaining stable for an extended range of $P_{\text {in }}$ and then changing directly over to chaos. Though the Feigenbaum route is known to be very sensitive to noise which might suppress the subsequent period. doublings, we interpret the observed behaviour to represent an independent route. We also observed a sequence of period triplings up to period 9 .

Intermittency means the occurrence of a signal which randomly altermates between two (or more) different dynamic states, e.g. laminar phases and irregular bursts. Three universal types of intermittency (I - III) have been discussed by Pomeau and Manneville [6] corresponding to the basically different ways how a fixed point of a 1D map can lose its stability in a local bifurcation. Both in the coincidence regime and for subsidiary absorption we observed various kinds of intermittency starting from a fixed point, a limit cycle, a 2-torus, or even alternating between different chaotic states. From analysing the distribution and scaling behaviour of the 'laminar' lengths, the observed signals could clearly be attributed to each of the universal types I, II or III or to crises [2]. It is interesting to note that the Pomeau-Manneville types are generally observed in parameter regimes where the system remains low-dimensional, whereas chaos-chaos intermittency occurs for higher dimensional states occurring in the coincidence regime. Very recently we identified a new type called 'on-off intermittency' which is based on a global symmetry-breaking bifurcation [7]. The underlying physical mechanism is probably that of the transitory excitation of an additional spin-wave mode through a 3-magnon process [2].

The analysis of the observed chaotic behaviour shows a significant difference between resonant and nonresonant pumping: In the coincidence regime we generally observe higher-dimensional chaos ('hyperchaos') with correlation dimensions [5] $D_{2} \simeq 7 \ldots 15$, whereas in subsidiary absorption low-dimensional ('marginal') chaos of dimension $D_{2} \simeq 2 \ldots 3$ is prevailing.

\section{CONTROLLING CHAOS}

Controlling chaos means to change the irregular auto-oscillations of a chaotic system into regular oscillations without drastically affecting the systern, just by making use of the internal system dynamics. Ott, Grebogi and Yorke (OGY) [8] have proposed a general way to achieve control by means of a feedback technique, where small time-dependent perturbations are made on one of the system parameters. Their algorithm is based on the idea that chaotic attractors are generally embedded in an infinite number of unstable periodic orbits (UPO). If the trajectory comes into the vicinity of an UPO, it approaches the orbit as long as the distance vector is located close to the siable manifold, and leaves it again in the direction of the unstable manifold. Since these processes evolve exponentially in time, they are rather slow in the vicinity of the orbit and can be affected by weak external perturbations. The practical use of such a control would be twofold: to suppress undesired irregularity and to select specific regular oscillations among a large number of possibilities by applying very small controlling power.

A different technique which also aims at the stabilization of an existing UPO was proposed by Pyragas [9]. It is based on a continuous time-delayed feedback which in the neighbourhood of the UPO may directly act as a proper controlling force to stabilize the regular motion on the orbit. We consider a nonlinear system which is described by the following set of differential equations: $\dot{x}=\boldsymbol{P}(\boldsymbol{x})$, where $\boldsymbol{x}=\left(x_{1}, \ldots, x_{\mathrm{N}}\right)$ are the system variables. At least one of them, $x_{1}$, should be accessible in experiment. The explicit knowledge of $\boldsymbol{P}$ is not required. The controlling scheme for an unstable periodic orbit $\xi$ consists in adding a forcing $F(t)=-K[x(t)-\xi(t)]$ which is directed towards the position of the UPO and acts as a negative feedback. Since the exact position of the UPO is $a$ priori unknown, $\boldsymbol{\xi}(t)$ is approximated by a part of the trajectory closer to it than the present position of $x(t)$. So, once the system has approached the UPO and is about to escape slowly, a proper correction is obtained by

$$
F(t)=-K[x(t)-x(t-\tau)]
$$

where $x(t-\tau)$ denotes the position of the trajectory one cycle before. The vanishing of the control signal $F(t)$ can be taken as a criterion for successful control. The method is especially well suited for periodically driven systems, since $\tau$ is exactly known in this case, but it can also be applied to autonomous systems.

By linear stability analysis of the uncontrolled and controlled system we have derived analytical expressions describing the efficiency of the control of real periodic orbits, its dependence on $K$ and $\tau$, and the critical and optimal $K$-value. It is important to note that anly orbits with a finite torsion, i.e. a nonvanishing imaginary part $\Omega_{\mathrm{c}}$ of the corresponding Floquet exponent, can be stabilized by the Pyragas method (for details see [10]).

The experimental set-up used for our control experiment is shown in Fig. 2 a. We chose the microwave input power as the feedback variable, which means that we applied a direct force on the uniform mode rather than a. parametric feedback. The control device consisted of a cascade of electronic delay lines with a limiting frequency of about $2 \mathrm{MHz}$. The device allowed to apply a control signal of the form $F(t)= \pm K\left[x_{1}(t)-\epsilon \cdot x_{1}(t-\tau)\right]$ with 
a)

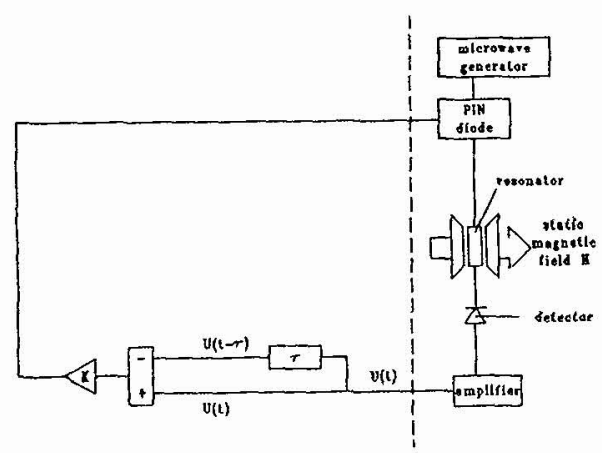

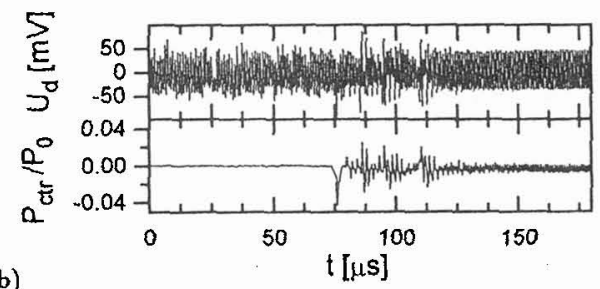

b)

b) Signal and control

Figure 2: Time-delayed fcedback control of a YIG sphere in subsidiary absorption. a) Experimental set-up
signal before and after switching on the control. The new regular orbit is embedded in the original attractor.

parameter ranges $K=0 \ldots 100, \epsilon=0 \ldots 2$, and $\tau=10 \ldots 7000 \mathrm{~ns}$. For conventional delayed feedback control $\epsilon$ has carefully to be adjusted to 1 , and $K$ and $\tau$ are set according to the conditions of the system. We succeeded in stabilizing orbits in the chaotic regime at $\omega_{\mathrm{p}} / 2 \pi=9.26 \mathrm{GHz}, H=1850 \mathrm{Oe}$, and $P_{\text {in }} \geq 10 \mathrm{~dB}$, but only after slightly detuning the parameter $\epsilon$ to a value smaller than 1 . The reason could be that the torsion frequency $\Omega_{c}$ was too small in comparison with the escape rate $\Lambda$ for the respective unstable orbit. However, by changing slightly the parameter $\epsilon$ the chaotic behaviour became regular, as shown in Fig. $2 \mathrm{~b}$. In a strict sense, we should not call this control, since for $\epsilon \neq 1$, the corresponding control signal cannot vanish asymptotically. Instead, the feedback is included to change the system and to form a new regular state. In the present case, however, this new orbit is very close to the previous unstable one.

\section{CONCLUSIONS}

A ferromagnetic sample externally driven by a strong microwave field represents an intriguing paradigm to study general properties of nonlinear dynamics. The reasons are manifold: (i) Magnetic systems represent intrinsically nonlinear systems, whose nonlinearities originate from well-known interactions. (ii) Their nonlinearities give rise to auto-oscillations, chaos and spatio-temporal pattern formation. (iii) The chaotic state can be controlled with the help of very weak external time-dependent perturbations. Nevertheless, in spin wave experiments we meet the problem that most of the interesting phenomena occur on rather inconvenient time and length scales. This makes the analysis of the chaotic state and the application of sophisticated control techniques sometimes rather tedious or even impossible. The time-delayed feedback method of Pyragas may be considered as an efficient compromise combining simplicity, universality, and robustness against noise with small controlling power and, thus, represents a promising method for practical use in fast technical systems.

This project of SFB 185 Nichtlineare Dynamik has been supported by special funds of the Deutsche Forschungsgemeinschaft.

\section{References}

[1] Wigen P.E. Ed., Nonlinear Phenomena and Chaos in Magnetic Materials (World Scientific, Singapore, 1994).

[2] Rödelsperger F., Chaos und Spinwelleninstabilitäten (Harri Deutsch, Frankfurt, 1994).

[3] Wiese G. and Benner H., Z. Phys. B 79 (1990) 119-131.

[4] Benner H., Rödelsperger F, and Wiese G., "Chaotic dynamics in spin-wave instabilities", Nonlinear Dynamics in Solids, H. Thomas Ed. (Springer, Berlin-Heidelberg, 1992) pp. 129-155.

[5] Schuster H.G., Deterministic Chaos (VCH, Weinheim, 1994).

[6] Pomeau Y. and Manneville P., (1980) Commun. Math. Phys. 74 (1980) 189.

[7] Rödelsperger F., Cenys A., and Benner H., Phys. Rev. Lett. 75 (195) 2594.

[8] Ott E., Grebogi C., and Yorke Y.A., Phys. Rev. Lett. 64 (1990) 1196.

[9] Pyragas K., Phys. Lett. A 170 (1992) 421.

[10]. W. Just, T. Bernard, M. Ostheimer, E. Reibold, H. Benner, to be published 